\title{
CONFLITOS ÉTICOS NO AMBIENTE ACADÊMICO DE MEDICINA
}

\author{
Bruno Araújo Novais Limal \\ Michael Sarmento Furtado I \\ João Manoel Lima de Barros Carvalho' \\ Paula Giovanna Costall \\ *Vilma Felipe Costa de Melo ${ }^{\text {II }}$
}

\section{RESUMO}

A ética no ambiente acadêmico deve ser continuamente levada em discussão. O comportamento antiético pode se apresentar de diversas formas e tem causas multifatoriais. $O$ presente artigo tem 0 objetivo de compreender e discutir o que é ética tomando como objeto de análise o comportamento ético e antiético dos estudantes de Medicina, assim como os reflexos atitudinais na atuação profissional desses indivíduos e suas repercussões. Foi elaborado, a partir de uma revisão integrativa, descritiva e exploratória entre 2012 e 2020, considerando as bases de dados da SciELO e Medline. Encontrou-se um total de 1029 artigos, sendo que apenas 13 desses se enquadravam nos critérios estabelecidos. Buscouse identificar a razão que leva os estudantes a antiética acadêmica, refletindo acerca dos conflitos éticos que permeiam tal temática. Concluiu-se, portanto, que a ética é primordial para a formação em Medicina, sendo necessário adotar estratégias que propiciem um espaço de discussão e reflexão coletiva, estimulando a valorização da questão ética e psicológica, promovendo mudanças que possam contribuir para a melhoria do padrão ético e moral nas relações acadêmicas.

PALAVRAS-CHAVE: Ética Médica. Estudantes de Medicina. Educação Médica.

Acadêmico de Medicina. Faculdade de Medicina Nova Esperança - FAMENE, Departamento de Medicina. I CEP: 58067-695, João Pessoa, Paraíba, Brasil. ORCID ID: 0000-0001-8645-7404; 0000-0002-9092-9520; 0000-0001-9688-7766 Bióloga. Mestranda, Universidade Federal da Pabaíba - UFPB, Programa de Pós Graduação de Filosofia. II CEP: 58397-000, João Pessoa, Paraíba, Brasil. ORCID ID: 0000-0001-5281-547X

Doutora em Filosofia. Docente das Faculdades Nova Esperança - FAMENE/FACENE, Programa de Pós Graduação III Mestrado Profissionalizante em Saúde da Família. *Email do autor correspondente: vilmelopsic@gmail.com CEP: 58067-695, João Pessoa, Paraíba, Brasil. ORCID ID: 0000-0001-5281-547X 


\section{INTRODUÇÃO}

A ética trata do comportamento moral de uma pessoa, ou grupo no mundo, sendo um dos pilares da filosofia e é, especialmente, relevante no trato de problemas que ultrapassam a regra e a norma. ${ }^{1}$ Assim, visto o perfil personalíssimo da ética, é dedutível que haja variação entre grupos sociais e constante evolução no decorrer da história. Portanto, seu enquadramento tradicional dá origem a várias vertentes, sendo uma delas a ética médica que, além de se ocupar com complexos debates de ordem social, tem seu foco em assuntos práticos relacionados a própria ciência médica na atenção aos pacientes e nos problemas que podem derivar dessa relação. ${ }^{2}$

O Conselho Federal de Medicina, através do Código de Ética Médica, no capítulo que trata dos Direitos Fundamentais, determina que o profissional é encarregado de cuidar e trabalhar pelo ético exercício da Medicina. Portanto, vemos a importância de abordar a ética médica durante o período da graduação. ${ }^{3}$ Para tanto, as Diretrizes Curriculares Nacionais, do curso de graduação em Medicina no Brasil, realçam a importância da formação ética e humanista para os estudantes da graduação de Medicina, visando desenvolver sua capacidade de atuação com responsabilidade social e compromisso na defesa da cidadania, da dignidade humana e da saúde integral do ser humano. ${ }^{3}$

Assim, apesar das habilidades para lidar com problemas no campo da ética serem inerentes a prática profissional médica, ainda são necessárias mais informações quanto aos conflitos éticos que ocorrem durante a graduação. ${ }^{4}$ Os médicos enfrentam problemas clínicos diariamente que, geralmente, são acompanhados por dilemas éticos os quais têm uma complexidade que vai além de normas e tecnicismos. ${ }^{2}$

A qualidade do ensino e o tempo investido na educação têm sido reconhecidos como os maiores fatores na promoção da competência de juízo moral, definida como a capacidade de tomar decisões que não contrariam a ética e de agir de acordo com tais julgamentos. Nesse sentido, a preocupação com a formação ética dos estudantes de Medicina tem implicações além do contexto da escola de Medicina. Na verdade, uma das principais razões pelas quais os sistemas de cuidados de saúde têm sido lentos na redução de eventos adversos evitáveis é a cultura longa e oculta de comportamentos desrespeitosos nos locais de cuidados a saúde, os quais levam também a erros médicos e aumento de custos. ${ }^{5}$

$O$ presente artigo tem o objetivo de compreender e discutir a perspectiva da ética no âmbito de comportamentos entre os estudantes de Medicina, assim como a reflexão do quanto um ensino de qualidade pode auxiliar no bom desempenho médico e garantir ao usuário um atendimento ético e humanizado.

\section{MATERIAL E MÉTODOS}

O presente trabalho foi elaborado, através de uma revisão integrativa, descritiva e exploratória, utilizando como estratégia de busca e fonte de informação consultadas nas bases de dados: Medical Literature Analysis and Retrieval System Online (Medline) e 
Scientific Electronic Library Online (SciELO), no período compreendido entre 2012 e 2020. A busca foi realizada com combinação de três descritores de busca (Descritores em Ciências da Saúde - DeCS/MeSH: Ética Médica. Estudantes de Medicina. Educação Médica).

Critérios de inclusão: 1. Artigos de revisão, estudos clínicos e estudos coorte; 2 . Estudos realizados no período de 2012 a 2020; 3. Foram incluídos como elegíveis estudos publicados e completos; 4. Artigos da área temática ciências da saúde, ética e ética médica.

Critérios de exclusão: 1. Artigos que não estavam de acordo com o período estabelecido

\section{RESULTADOS E DISCUSSÃO}

O estudo da ética médica, junto ao conhecimento técnico, deve ser a prioridade para os estudantes da graduação médica, já que futuramente estarão atuando com pessoas. Desta forma, o estudante deve aprender a enxergar os pacientes como seres humanos que precisam ser respeitados e não tratados como objeto de estudo, ou fonte de informação. Lamentavelmente, nem sempre há respeito quanto às necessidades dos pacientes, durante a prática profissional, decorrente da objetificação do ser humano. Desse modo, se faz necessária a consolidação do consentimento em âmbito acadêmico. Tal princípio ético deve ser respeitado, com o discernimento profissional que qualquer intervenção na área médica - seja de caráter preventivo, diagnóstico ou terapêutico - só poderá ser realizada com o prévio consentimento livre e esclarecido do sujeito. $^{3}$

A Medicina corresponde a uma área em que os profissionais devem carregar consigo toda uma bagagem ética que atua com as particularidades das pessoas. Porém, de 2012 a 2020; 2. Capítulos de editoriais, livros, relatos de caso, carta, comunicação rápida, editorial e artigo-comentário

Foram encontrados um total de 1029 artigos, sendo escolhidos 146, após processo de seleção com critérios de inclusão e exclusão propostos. Nos textos, foi feita análise do título a fim de verificar aqueles que estavam de acordo com a temática do estudo. 55 artigos foram selecionados para análise dos resumos, dos quais 13 se enquadravam nos critérios estabelecidos e efetivamente lidos por completo. os estudantes de Medicina são exaustivamente treinados para atuarem como profissionais técnicos, enquanto a questão moral eética ainda épouco trabalhada. A extrema competitividade que se observa desde a instituição até a imersão dos estudantes no mercado de trabalho é uma das razões pelas quais os acadêmicos valorizam tanto a qualificação técnica, já que essa é extremamente cobrada e muitos resolvem o problema moral negando-o ou confundindo-o com um assunto de natureza técnica. ${ }^{4}$

A ética médica busca unir as normas de comportamento adequado ao campo de cuidado ao doente, como uma entidade concreta devendo ser estimulada a reflexão de uma conduta apropriada desde a formação. Para tal, as faculdades médicas e seus docentes devem ter conhecimento das dificuldades enfrentadas pelos estudantes, relacionadas não apenas com questões curriculares e pedagógicas, mas também com a formação da identidade médica. ${ }^{5}$

Quando se trata de relatar comportamento antiético, no que se refere 
à questão moral, pesquisa mostra que há um dilema enfrentado pelos estudantes de Medicina, embora os acadêmicos afirmem que denunciar tais atos seja correto, poucos o fazem devido ao medo das possíveis consequências e repercussões tomadas pela mesma. Além disso, não consideram como responsabilidade deles denunciarem os comportamentos antiéticos praticados por colegas. ${ }^{6}$ Sendo necessário refletir, ao longo da formação, sobre o ser humano que é o médico, para auxiliar o aluno a entender e se adaptar as novas situações. ${ }^{4}$

Pesquisa em Santa Catarina alega que as mudanças atitudinais que ocorrem durante o processo de formação médica derivam de modificações na concepção do estudante acerca do "bom médico" e das expectativas em relação ao curso. Portanto, inicialmente os estudantes idealizam o curso de uma forma voltada a práticas humanizadas, mas passam a questionar a dinâmica do processo de formação por volta do terceiro ou quarto ano; no quinto e sexto ano, evidenciam certo desencanto com o processo vivenciado. ${ }^{5}$ Pesquisadores de Faculdade de Medicina em São Paulo defendem que a forma como os alunos são recebidos, acolhidos e orientados durante a vida acadêmica irá influenciar no grau de satisfação do curso escolhido e na qualidade do profissional que se tornará. ${ }^{4}$

Estudo realizado em Minas Gerais encontrou como as principais atitudes de estudantes que os levam a comportamentos antiéticos no âmbito acadêmico são: "cola", obtendo respostas através de outro colega; plágio, fazendo uso de ideias, figuras ou textos, sem a autorização do autor; adulteração, falsificação dos relatórios de aulas práticas ou pesquisas em campo; múltiplas submissões, na qual o aluno entrega um trabalho com pequenas modificações de outro texto que já tenha sido entregue; adulteração, simular a assinatura de outro aluno; dissimulação, em que o aluno ao perceber um erro do professor que lhe seja favorável e simula não perceber o equívoco para se beneficiar; mentira, o aluno apela para o lado emocional e tenta influenciar o professor a mudar data de provas ou algum benefício nas notas.?

A instituição de ensino com formação profissional de maior sucesso será a que, além da técnica, trabalha para enraizar uma cultura de integridade, orientando e exigindo honestidade do aluno. Deve ser uma prática contínua e dialogada em toda comunidade acadêmica, em todos os estágios do curso e por todos os professores, uma vez que eles precisam ser treinados continuamente pela instituição. ${ }^{5}$

Diante das diversas formas de atitudes antiéticas acadêmicas citadas, pode-se inferir que há todo um processo esquemático entre os alunos e que a fraude das mais variadas formas se tornou parte da cultura de alguns estudantes. ${ }^{6}$ Ainda assim, grande parte dos estudantes consideram erradas tais atividades, embora não acreditem que seja de responsabilidade deles relatar a ausência ética de colegas. Sendo que esta característica se encontra mais presente nas mulheres. ${ }^{8}$ Reforça-se que, os educadores em Medicina têm a obrigação de examinar atributos e determinantes da integridade acadêmica dos estudantes, durante a graduação.

Porém, atitudes antiéticas, durante a graduação, podem ser derivadas de uma fase acadêmica profundamente estressante devido à constante presença de fatores causadores de tensão e suas consequências. Investigações determinam que o tempo demandado para os estudos, pressão para aprender, exigência de alto rendimento, volume de informações, falta de tempo para atividades sociais, contato com pessoas doentes e com a morte são situações 
que causam um desgaste a saúde mental dos estudantes de Medicina, propiciando-os a falta de reflexão sobre as consequências de seu comportamento e interferindo na capacidade de desempenhar atividades do dia a dia como trabalhar ou estudar, prejudicando diretamente o processo de aprendizagem e formação. ${ }^{9}$

Sendo assim, como exemplo de tal formação profissional, percebem-se falhas éticas no convívio dos médicos nas redes sociais. A evolução tecnológica e a divulgação do conhecimento marcam acentuadamente a atualidade, podendo ser utilizada como instrumentos importantes no tratamento de doenças e melhoramento da saúde. Porém, há limites para o compartilhamento de informações por pessoas da área médica em suas redes sociais que devem ser respeitados. A privacidade do paciente, assim como a confidencialidade de seus dados devem ser tratados em sigilo profissional. As informações dos pacientes não podem ser utilizadas para fins que não foram consentidos pelo paciente. Para além disso, devem estar em concordância com o Código Internacional dos Direitos Humanos. Portanto, é importante que os profissionais médicos estejam atentos a essas atitudes dos estudantes, para orientá-los quanto a quebra da ética e forma correta de uso das redes sociais. ${ }^{10}$

A quebra do sigilo profissional é uma prática considerada como uma infração ética, com previsão de penalidades pelo Conselho Federal de Medicina e a exposição de fotos ou documentos do paciente pode constrangêlo levando a uma perda de confiança no profissional médico. ${ }^{7}$

A saúde, principalmente mental, dos estudantes de medicina há muito tem sido objeto de atenção e pesquisas no meio acadêmico. $O$ estresse e problemas mentais, como a ansiedade e a depressão, são descritos nessa população de estudantes e associados ao baixo rendimento acadêmico. A carga horária dos estudantes na faculdade é extensa, distribuída em atividades de ensino, pesquisa e extensão. $O$ estresse decorre da dificuldade em administrar o tempo exigido pelas diferentes disciplinas e o lazer. Soma-se a isso o contato com os pacientes e com a morte, bem como a competitividade entre os colegas, que se acirra com a valorização do desempenho acadêmico durante a graduação, perdurando até o processo seletivo para a entrada na residência. ${ }^{11}$

A necessidade de educação em ética médica é sustentada por diversos estudos científicos e organizações nacionais e internacionais como, por exemplo, a Organizações Internacionais de Ciências Médicas (CIOMS). A principal razão para essa necessidade de educação durante o período de graduação é o fato de os profissionais médicos estarem lidando com seres humanos, sendo a relação médico-paciente uma base para essa interação que envolve confiança e responsabilidade. Há diversas formas para a educação em ética médica, destacando as palestras que servem como fonte informativa quanto aos princípios éticos básicos. A prática educacional é outra forma de aprendizagem, à qual é realizada por meio de atividades desenvolvidas em pequenos grupos de estudantes que discutem cenários de conflito ético baseados em casos reais com o auxílio de um professor. ${ }^{12}$

Com a finalidade de ampliar a dimensão ética do aluno, a instituição de formação médica deve refletir sobre o seu papel no acolhimento e acompanhamento dos alunos por todo o processo de aprendizagem, ao longo do curso, de forma que a valorização da questão ética e psicológica se torne mais presente tanto no âmbito das salas de ensino como nos hospitais. ${ }^{13}$ 


\title{
CONSIDERAÇÕES FINAIS
}

\author{
$O$ estudo atual permitiu refletir acerca \\ dos conflitos éticos, tanto por estudantes \\ da graduação como por profissionais \\ da área médica. Dentre várias, temos o \\ compartilhamento de informações de pacientes \\ nas redes sociais, caracterizando quebra da \\ privacidade e do anonimato inerentes ao ato \\ médico e a competividade interna entre os \\ colegas de curso, que está representada pelo \\ desempenho acadêmico, leva a ações antiéticas
}

\begin{abstract}
e imorais. Com o avanço do curso, temos uma regressão quanto ao comportamento ético dos estudantes perante os pacientes. Portanto, adotar estratégias que propiciem um espaço de discussão e reflexão coletiva acerca dos problemas ao longo da graduação é uma forma de resolução ou, pelo menos, minimização das relações de conflitos éticos pelas quais passam os estudantes de Medicina, assim como a atuação de forma ética.
\end{abstract}

\section{ETHICAL CONFLICTS IN THE ACADEMIC MEDICINE ENVIRONMENT}

\begin{abstract}
The ethics in the academic environment must be continually taken in to discussion. Academic unethical behavior can present itself in various ways and has multifactorial causes. The present article aims raise a discussion about academic unethical in medical courses, its types, and its possible consequences in the main generating elements of this phenomenon. This article was elaborated from an integrative, descriptive, and exploratory review, between 2012 and 2020, considering the databases from the SciELO and Medline. A total of 1029 articles were found, but only 13 articles meet the specified criteria. The aim of this research was to identify the reason which leads students to an unethical behavior in the academy, reflecting on the ethical conflicts that permeate this subject. Therefore, it was concluded that ethics is primary for medical training, being necessary to adopt strategies that provide a space for discussion and collective evaluation, stimulating the appreciation of the ethical question and psychological, promoting changes that may contribute to an improvement in the ethical standard and moral in academic relations.
\end{abstract}

KEYWORDS: Medical Ethics. Medical Students. Medical Education. 


\section{REFERÊNCIAS}

1. Utz, K. O "Progresso na Consciência da Liberdade": Um Aspecto Ético da Filosofia da História de Hegel. Ethic@ - Florianópolis, Santa Catarina, Brasil. Jul. 2015; 14 (1):82-103.

2. Rojas OA, Libia LC. Ética, bioética e ética médica. Rev. Chil. Enferm. Respir. Jun. 2014; 30 (2):9194.

3. Menezes MM, Maia LC, Rocha CU, Sampaio CA, Costa SM. Conflitos Éticos Vivenciados por Estudantes de Medicina. Rev. Bras. Educ. Med. 2017; 41 (1):69-162.

4. Feitosa HN, Rego S, Bataglia P, Rego G, Nunes R. Competência de juízo moral dos estudantes de medicina: um estudo piloto. Rev. Bras. Educ. Med., Rio de Janeiro. Mar. 2013; 37 (1): 5-14.

5. Miranda SM, Pires MMS, Nassar SM, Silva CAJ. Mudança de atitudes dos estudantes durante o curso de medicina: um estudo de coorte. Rev. Bras. Educ. Med. 2012; 36 (2):212-22.

6. Ghias K, Lakho GR, Asim H, Azam IS, Saeed SA. Self-reported attitudes and behaviours of medical students in Pakistan regarding academic misconduct: a cross-sectional study. BMC Med Ethics. May. 2014; 29, 15:43.

7. Sousa RN, Conti VK, Salles AA, Mussel ICR. Desonestidade acadêmica: reflexos na formação ética dos profissionais de saúde. Rev. Bioét. Dez.
2016; $24(3): 459-68$.

8. Tanaka MM, Furlan LL, Branco LM, Valerio NI. Adaptação de Alunos de Medicina em Anos Iniciais da Formação. Rev. Bras. Educ. Med. 2016; 40 (4):663-68.

9. Bampi LNS, Baraldi S, Guilhem D, Araújo MP, Campos ACO. Qualidade de vida de estudantes de medicina da Universidade de Brasília. Rev. Bras. Educ. Méd. 2013; 37 (2):217-25.

10. Souza ES, Lorena SB, Ferreira CCG, Amorim AFC, Peter JVS. Ética e Profissionalismo nas Redes Sociais: Comportamentos On-Line de Estudantes de Medicina. Rev. Bras. Educ. Med., Rio de Janeiro. Set. 2017; 41 (3):412-23.

11. Chazan ACS, Campos MR. Qualidade de vida de estudantes de medicina medida pelo WHOQOL-bref - UERJ, 2010. Rev. Bras. Educ. Med. 2013; 37 (3):376-84.

12. Bilgin AC, Timbil S, Guvercin CH, Ozan S, Semin S. Preclinical Students' Views on Medical Ethics Education: A Focus Group Study in Turkey. Acta bioeth. Santiago. Jun. 2018; 24 (1):105-15.

13. Chazan ACS, Campos MR, Portugal FB. Qualidade de vida de estudantes de medicina da UERJ por meio do Whoqol-bref: uma abordagem multivariada. Ciênc. Saúde Coletiva. Fev. 2015; 20 (2):547-56. 